Journal of Advanced Research in Fluid Mechanics and Thermal Sciences

Journal homepage: www.akademiabaru.com/arfmts.html ISSN: 2289-7879

\title{
Mathematical Analysis of Unsteady Solute Dispersion with Chemical Reaction Through a Stenosed Artery
}

\author{
Nurul Aini Jaafar ${ }^{1,}{ }^{*}$, Siti NurulAifa Mohd ZainulAbidin ${ }^{1}$, Zuhaila Ismail $^{1}$, Ahmad Qushairi Mohamad $^{1}$ \\ 1 Department of Mathematical Sciences, Faculty of Science, Universiti Teknologi Malaysia, 81310 UTM Johor, Johor Bahru, Malaysia
}

\section{ARTICLE INFO}

\section{Article history:}

Received 2 June 2021

Received in revised form 20 July 2021

Accepted 25 July 2021

Available online 20 August 2021

\section{Keywords:}

Blood flow; Dispersion function; Generalized dispersion model; HerschelBulkley; Non-Newtonian fluids; Stenosed artery

\section{ABSTRACT}

One major kind of arterial disease in blood flow that attracted many researchers is arterial stenosis. Arterial stenosis occurs when a lumen of artery is narrowed by the accumulation of fats, cholesterols and lipids plaques at the inner layer of the wall of an artery. To treat this arterial disease, the drug (solute) is injected into the blood vessels. Injection of the drug into the blood vessel cause the occurrence of chemical reaction between the drug and blood proteins and it affects the effectiveness of the solute transportation in blood flow. Hence, this study examines the unsteady dispersion of solute with the influence of chemical reaction and stenosis height through a very narrow artery with a cosine-curved stenosis. The blood is treating as Herschel-Bulkley (H-B) fluid. The momentum and constitutive equations are solved analytically to gain velocity of $\mathrm{H}-\mathrm{B}$ blood flow. The convective-diffusion equation is solved by applying the generalized dispersion model to gain the dispersion function of solute. The influence of chemical reaction, power-law index, plug flow radius and stenosis height on the solute dispersion process is investigated. The results are validated with the previous solution without the effect of chemical reaction and stenosis. The results showed a good conformity between the two solutions. An increase in the chemical reaction coefficient, stenosis height, power-law index and plug flow radius reduces the dispersion function. It is observed that the solute dispersion in blood flow is affected by chemical reaction and stenosis height. $\mathrm{H}-\mathrm{B}$ fluid is an appropriate fluid to investigate the blood velocity and transportation of the drug in blood flow to the targeted stenosed region through a very narrow artery for the treatment of arterial diseases. The results of the present study can potentially be used to predict the changes of blood flow behavior and dispersion process in blood flow.

\section{Introduction}

According to the statistics issued by World Health Organization (WHO), the cardiovascular diseases such as ischaemic heart disease and stroke were the major cause of death in the world. The ischaemic heart disease, responsible for 16 percent of the total deaths in the world, is the world's greatest killer. The ischaemic heart disease also known as coronary artery diseases is a disease in which blood flow is limited or decreased due to narrowing of the arteries. As a result, less blood and

\footnotetext{
* Corresponding author.

E-mail address: nurulaini.jaafar@utm.my
}

https://doi.org/10.37934/arfmts.86.2.5673 
oxygen enter the heart muscles, resulting in a heart attack. The most common cause of ischemic heart disease is atherosclerosis or called stenosis, in which the coronary arteries narrow due to a progressive accumulation of fatty material caused by the build-up of fats, cholesterol and other substances within the inner wall of artery lumen. A thickening of the stenosis can lead to the narrowing of the diameter of inside artery and can block the blood flow to organs and tissues. The severe stenosis can cause the cardiovascular diseases. The flow of blood in a stenosed artery is very evident and distinct from normal arteries [1]. Thus, it is important to study the blood flow through a stenosed artery in order to examine the blood rheology.

The solute transportation in blood flow was studied and very important in several research, such as transferring drugs and toxin in the physiological system [2]. For example, the drug was directing inject into artery to cure diseases such as cancer and more. The injection of drugs can be therapeutic at low concentration and toxic at the high concentration of drugs. Thus, it is essential to examine the rate of dispersion to obtain the effectiveness of the drug in blood flow. The problem of solute dispersion process in blood flow is getting more important and it has more widely studied by researchers. Investigating the solute dispersion in blood flow is crucial for better treatment of cardiovascular diseases. The first person who studied the solute dispersion through a straight tube for Newtonian fluid was Taylor [3]. In Taylor's contribution, he explained the combination of the velocity movement and lateral molecular diffusion over the cross-section. Aris [4] executing the method of moments reported that the dispersion theory of Taylor is only valid when $D_{\text {eff }} \geq D_{m}$ and thereby enhanced the dispersion theory by including the effect of axial molecular diffusion $D_{\text {eff }}=D_{m}+a^{2} w_{m}^{2} / 48 D_{m}$. Ananthakrishnan et al., [5] studied the diffusion solute of axial and radial molecular to get the numerical and empirical results. Gill and Sankarasubramanian [6] enhanced the study on solute dispersion by formulating the generalized dispersion model (GDM) to obtain the entire process of solute dispersion using the/ derivative series expansion.

In the previous study, many researchers analyzed the Newtonian fluid and certain non-Newtonian like Bingham, power-law as well as Casson fluids to investigate the problems involving the blood flow and solute dispersion that occurs in the bloodstream. Dash et al., [7] examined the unsteady solute dispersion in steady flow of Casson fluid using GDM. Rana and Murthy [8] examined the unsteady dispersion of a solute in a small vessel in unsteady flow when the solute absorption at the vessel wall occurred and not occurred by considering two-phase models of Casson fluid together with powerlaw using GDM. Rana and Murthy [9] extended the study to investigate the impact of yield stress on the transportation of solute for unsteady flow of two-phase Casson fluid.

$\mathrm{H}-\mathrm{B}$ fluid is one of the useful and important fluid models to investigate the blood viscosity and yield stress. In the previous study, it is seen that the influences of stenosis on the dispersion of a solute with the effect of chemical reaction in the bloodstream do not receive much attention to many researchers. Jaafar et al., [2] investigated the influence of chemical reaction on the steady dispersion of solute in blood flow of Casson fluid in blood flow in a straight pipe and a flat plate. Jaafar [10] extended the study to investigate the transportation of solute in a straight pipe and a flat plate in blood flow of H-B fluid for the unsteady solute dispersion using GDM with the effect of chemical reaction. Rana and Murthy [11] studied the mathematical modelling of unsteady solute dispersion in micropolar-Newtonian blood flow of two fluid with bulk reaction using GDM. Jaafar et al., [12] analysed the effect of chemical reaction on solute dispersion in a blood flow of Casson fluid fluid for the unsteady solute dispersion using GDM. The effect of stenosis on the solute dispersion with the effect of chemical reaction has been not studied by anyone so far.

Thus, to the best of our knowledge, this study investigates the dispersion of solute in the blood flow of $\mathrm{H}-\mathrm{B}$ fluid with the effect of stenosis and chemical reaction. The momentum and constitutive 
equations have been solved analytically to obtain the velocity of a H-B fluid. The obtained velocity has been used to get the unsteady dispersion coefficient by solving the convective-diffusion equation analytically using GDM to obtain the effective axial diffusivity in the presence of chemical reaction through a stenosed artery. The study may help to understand the physiological processes of the injection of drug in the bloodstream and predict the effectiveness of the drug dispersion when the chemical reaction exists in the stenosed artery.

\section{Mathematical Formulation}

Consider the flow of blood assumed as viscous incompressible fluid through a circular pipe in a laminar, continuous, axisymmetric, and fully formed unidirectional flow in the axial direction, treating blood as H-B fluid. The pipe flow geometry describes the artery with the presence of chemical reaction are shown in Figure 1 , where $\bar{u}$ is the axial velocity of the fluid, $R_{0}$ is the radius of pipe, $\bar{r}$ and $\bar{z}$ are the coordinates in the radial and axial directions, respectively, $\bar{r}_{c}$ is the plug core radius, $\bar{\delta}$ is the height of stenosis, $\bar{l}_{0}$ is the length of a stenosis, $\bar{d}$ is the stenosis location, $\bar{L}$ is the artery length and $\bar{R}(\bar{z})$ is the stenotic artery radius.

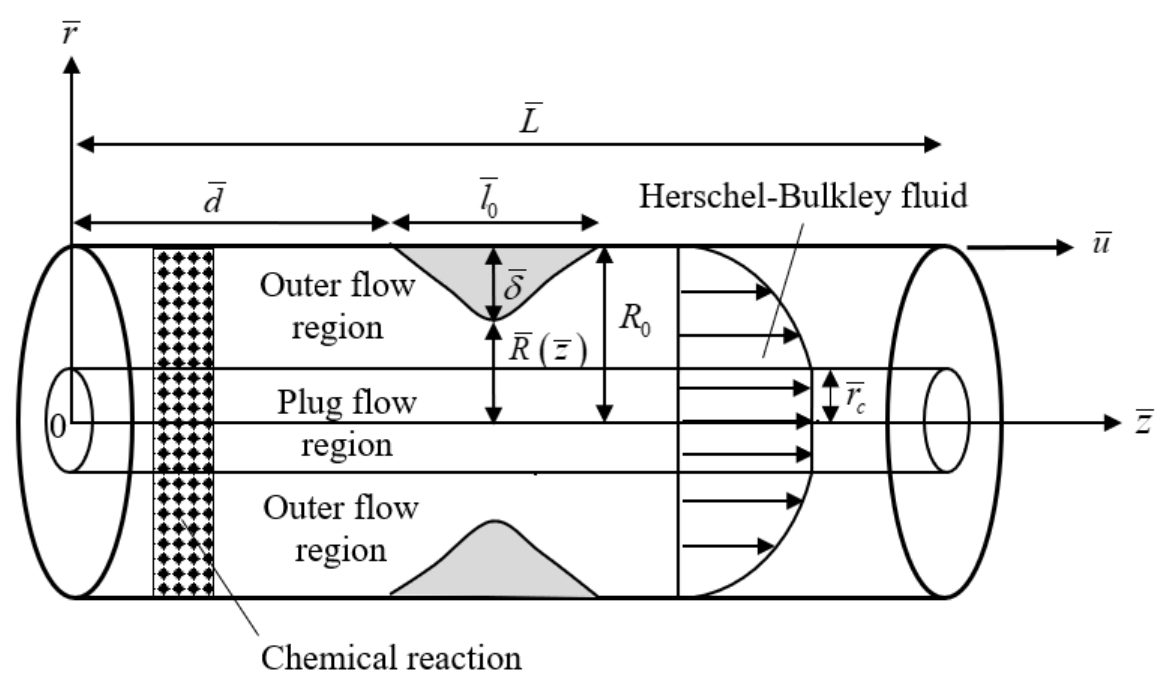

Fig. 1. The blood flow geometry in a stenosed artery

For a steady slow flow of viscous incompressible fluid, consider the axial and radial components of the momentum equations as follows:

$\frac{d \bar{p}}{d \bar{z}}=-\frac{1}{\bar{r}} \frac{d}{d \bar{r}}(\bar{r} \bar{\tau})$

$\frac{d \bar{p}}{d \bar{r}}=0$

where $\bar{p}$ is the pressure and $\bar{\tau}$ is the shear stress. The pressure in Eq. (1) and Eq. (2) varies only in the axial direction and constant in the radial direction. The constitutive equation of $\mathrm{H}-\mathrm{B}$ fluid model is given by 
$\frac{d \bar{u}}{d \bar{r}}= \begin{cases}-\frac{1}{\bar{\eta}_{H}}\left(\bar{\tau}-\bar{\tau}_{y}\right)^{n} & \text { if } \bar{\tau}>\bar{\tau}_{y} \text { and } \bar{r}_{p} \leq \bar{r} \leq \bar{R}(\bar{z}), \\ 0 & \text { if } \bar{\tau} \leq \bar{\tau}_{y} \text { and } 0 \leq \bar{r} \leq \bar{r}_{p},\end{cases}$

where $\bar{\eta}_{H}$ denotes the viscosity coefficient of $\mathrm{H}$-B fluid, $n$ denotes the power-law index, $\bar{\tau}_{y}$ is the yield stress and

$$
\bar{R}(\bar{z})=\left\{\begin{array}{lr}
R_{0}-\frac{\bar{\delta}}{2}\left[1+\cos \left(\frac{2 \pi}{\bar{l}_{0}}\left(\bar{z}-\bar{d}-\frac{\overline{l_{0}}}{2}\right)\right)\right], \bar{d} \leq \bar{z} \leq \bar{d}+\bar{l}_{0}, \\
R_{0}, \quad \text { otherwise. }
\end{array}\right.
$$

According to Eq. (3), normal shear flow occurs when shear stress exceeds yield stress, while unshear flow (plug flow or solid-like flow) occurs when shear stress is equal to or less than yield stress. The following boundary conditions can be used to solve Eq. (1) and Eq. (3) for the unknowns' shear stress and velocity

$\bar{\tau}$ at $\bar{r}=0$,

$\bar{u}=0$ at $\bar{r}=\bar{R}(\bar{z})$.

The convective-diffusion equation with the chemical reaction in simplified form that describes the solute dispersion process undergoes with chemical reaction is given as follows

$$
\frac{\partial \bar{C}}{\partial \bar{t}}+\bar{u} \frac{\partial \bar{C}}{\partial \bar{z}}=\bar{D}_{m}\left[\bar{L}^{2}+\frac{\partial^{2}}{\partial \bar{z}^{2}}\right] \bar{C}-\bar{\beta} \bar{C}
$$

where $\bar{C}$ is the solute concentration, $\bar{t}$ is the time variable, $\bar{D}_{m}$ is the coefficient of molecular diffusivity, $\bar{\beta}$ is the chemical reaction rate and

$$
\bar{L}^{2}=\frac{1}{\bar{r}} \frac{\partial}{\partial \bar{r}}\left(\bar{r} \frac{\partial}{\partial \bar{r}}\right)
$$

The initial and boundary conditions of the Eq. (7) are respectively given by

$$
\bar{C}(\bar{r}, \bar{z}, 0)= \begin{cases}\bar{C}_{0}, & \text { if }|\bar{z}| \leq \frac{\bar{z}_{s}}{2}, \\ 0, & \text { if }|\bar{z}|>\frac{\bar{z}_{s}}{2},\end{cases}
$$

where $\bar{C}_{0}$ is the reference concentration.

$\bar{C}(\bar{r}, \infty, \bar{t})=0$, 


$$
\frac{\partial \bar{C}}{\partial \bar{r}}(0, \bar{z}, \bar{t})=0=\frac{\partial \bar{C}}{\partial \bar{r}}(\bar{R}(\bar{z}), \bar{z}, \bar{t})
$$

\subsection{Non-dimensional Variables}

Consider the following non-dimensional variables

$$
\begin{aligned}
& u=\frac{\bar{u}}{\bar{u}_{0}}, u_{+}=\frac{\bar{u}_{+}}{\bar{u}_{0}}, u_{-}=\frac{\bar{u}_{-}}{\bar{u}_{0}}, u_{m}=\frac{\bar{u}_{m}}{\bar{u}_{0}}, r=\frac{\bar{r}}{R_{0}}, r_{p}=\frac{\bar{r}_{p}}{R_{0}}, \tau=\frac{\bar{\tau}}{\left(\eta \bar{u}_{0} / R_{0}\right)^{1 / n}}, \tau_{y}=\frac{\bar{\tau}_{y}}{\left(\eta \bar{u}_{0} / R_{0}\right)^{1 / n}}, \\
& R(z)=\frac{\bar{R}(\bar{z})}{R_{0}}, \delta=\frac{\bar{\delta}}{R_{0}}, z=\frac{\bar{z}}{R_{0}}, z=\frac{\bar{D}_{m} \bar{z}}{R_{0}^{2} \bar{u}_{0}}, z_{s}=\frac{\bar{D}_{m} \bar{z}_{s}}{R_{0}^{2} \bar{u}_{0}}, C=\frac{\bar{C}}{\bar{C}_{0}}, t=\frac{\bar{D}_{m} \bar{t}}{R_{0}^{2}}, \beta=\sqrt{\frac{R_{0}^{2} \bar{R}}{\bar{D}_{m}}}, P e=\frac{\bar{R}(\bar{z}) \bar{u}_{0}}{\bar{D}_{m}},
\end{aligned}
$$

where

$$
\bar{u}_{0}=\frac{\bar{R}(\bar{z})^{n+1}}{(n+1) \bar{\eta}}\left(-\frac{1}{2} \frac{d \bar{p}}{d \bar{z}}\right)^{n}
$$

is the characteristic velocity, $u$ is the velocity, $u_{+}, u_{-}$is the non-plug and plug flow velocity, $u_{m}$ is the average velocity, $C$ is the solute concentration, $\beta$ is the rate of chemical reaction, $P e$ is the modified Peclet number, $r$ is the radial distance, $r_{p}$ is the radius of the plug core field, $\delta$ is the stenosis height, $z$ is the longitudinal distance, $z_{s}$ is the solute length and $t$ is the time in non-dimensional form. $\bar{\delta} / R_{0}$ in Eq. (12) is known as stenosis severity [13]. Using the non-dimensional variables (12), the Eq. (1)(4) becomes

$\frac{d p}{d z}=-\frac{1}{r} \frac{d}{d r}(r \tau)$,

$\frac{d p}{d r}=0$

$\frac{d u}{d r}=\left\{\begin{array}{cr}-\left(\tau-\tau_{y}\right)^{n} & \text { if } \tau>\tau_{y} \text { and } r_{p} \leq r \leq R(z), \\ 0 & \text { if } \tau \leq \tau_{y} \text { and } 0 \leq r \leq r_{p},\end{array}\right.$

$R(z)=\left\{\begin{array}{lr}1-\frac{\delta}{2}\left[1+\cos \left(\frac{2 \pi}{l_{0}}\left(z-d-\frac{l_{0}}{2}\right)\right)\right], d \leq z \leq d+l_{0} \\ 1, & \text { otherwise }\end{array}\right.$

and boundary conditions (5) and (6) becomes

$\tau$ at $r=0$,

$u=0$ at $r=R(z)$. 
The non-dimensional form of the unsteady convective diffusion equation is given by applying the non-dimensional variables in Eq. (7) as follows

$$
\frac{\partial C}{\partial t}+u \frac{\partial C}{\partial z}=\left[L^{2}+\frac{1}{P e^{2}} \frac{\partial^{2}}{\partial z^{2}}\right] C-\beta^{2} C
$$

where

$$
L^{2}=\frac{1}{r} \frac{\partial}{\partial r}\left(r \frac{\partial}{\partial r}\right)
$$

and

$$
P e=\frac{\bar{a} \bar{u}_{0}}{\bar{D}_{m}}
$$

is the Péclet number given by Dash et al., [7]. Then, non-dimensional form of the initial and boundary conditions for the concentration of the solute (9)-(11) are

$$
C(r, z, 0)= \begin{cases}1, & \text { if }|z| \leq \frac{z_{s}}{2}, \\ 0, & \text { if }|z|>\frac{z_{s}}{2},\end{cases}
$$

$C(r, \infty, t)=0$,

$$
\frac{\partial C}{\partial r}(0, z, t)=0=\frac{\partial C}{\partial r}(R(z), z, t)
$$

\subsection{Method of Solution}

Integrating Eq. (14) with respect to $r$, and then using boundary condition (18), the shear stress is obtained in non-dimensional form as $\tau=2 r$. Then, by replacing $r$ to $r_{p}$, the expression for the yield stress in non-dimensional form is $\tau_{y}=2 r_{p}$. Two terms of the binomial series approximation have been used in Eq. (16) and applying the $\tau=2 r, \tau_{y}=2 r_{p}$ and boundary condition (19) in the resulting equation, the expression for the velocity of $\mathrm{H}$-B fluid in the outer flow (shear flow) region is as follows

$u_{+}(r)=\left(1-\frac{r^{n+1}}{R^{n+1}(z)}\right)-(n+1) \frac{r_{p}}{R(z)}\left(1-\frac{r^{n}}{R^{n}(z)}\right)+\frac{n(n+1)}{2} \frac{r_{p}^{2}}{R^{2}(z)}\left(1-\frac{r^{n-1}}{R^{n-1}(z)}\right)$ if $r_{p} \leq r \leq R(z)$.

The expression for the velocity of H-B fluid in the plug flow region is obtained from Eq. (26) by evaluating it at $r=r_{p}$ and is given as follows 


$$
u_{-}\left(r_{p}\right)=1-(n+1) \frac{r_{p}}{R(z)}+\frac{n(n+1)}{2} \frac{r_{p}^{2}}{R^{2}(z)}-\frac{n(n-1)}{2} \frac{r_{p}^{n+1}}{R^{n+1}(z)} \text { if } 0 \leq r \leq r_{p} .
$$

The mean velocity of blood flow in a cosine-curved stenosed artery for $\mathrm{H}-\mathrm{B}$ is as follows:

$$
u_{m}=\frac{(n+1)}{(n+3)}\left[1-\frac{n(n+3)}{(n+2)} \frac{r_{p}}{R(z)}+\frac{n(n+3)(n-1)}{2(n+1)} \frac{r_{p}^{2}}{R^{2}(z)}-\frac{\left(n^{4}+2 n^{3}-5 n^{2}-6 n+4\right)}{2(n+1)(n+2)} \frac{r_{p}^{n+3}}{R^{n+3}(z)}\right]
$$

Consider a new coordinate system $\left(r, z_{1}, t\right)$ with a new axial coordinate $z_{1}$ for solute convection over a plane moving with the fluid's average velocity $u_{m}$, so that the axis moves with the fluid's mean velocity. A new axial coordinate $z_{1}$ is defined as

$$
z_{1}=z-u_{m} t
$$

Using the approach of Gill and Sankarasubramanian [6], let us assume the solution of Eq. (20) in a derivative series expansion involving $\partial^{j} C_{m} / \partial z_{1}^{j}$, as follows:

$$
C\left(r, z_{1}, t\right)=C_{m}\left(z_{1}, t\right)+\sum_{j=1}^{\infty} f_{j}(r, t) \frac{\partial^{j} C_{m}\left(z_{1}, t\right)}{\partial z_{1}^{j}}
$$

where

$$
C_{m}\left(z_{1}, t\right)=2 \int_{0}^{R(z)} C\left(r, z_{1}, t\right) r d r
$$

is the mean concentration of the solute over a cross section and $f_{j}(r, t)$ is the dispersion function associated with $\partial^{j} C_{m} / \partial z_{1}^{j}$, where $f_{0}(r, t)=1$ following the initial condition (23). Substituting Eq. (30) in Eq. (20), one can obtain

$$
\begin{aligned}
& \frac{\partial C_{m}}{\partial t}+\left(u-u_{m}\right) \frac{\partial C_{m}}{\partial z_{1}}-\frac{1}{P e^{2}} \frac{\partial^{2} C_{m}}{\partial z_{1}^{2}}+\alpha^{2} C_{m}+\sum_{j=1}^{\infty}\left[\left(\frac{\partial f_{j}}{\partial t}-L^{2} f_{j}\right) \frac{\partial^{j} C_{m}}{\partial z_{1}^{j}}+\left(u-u_{m}\right) f_{j} \frac{\partial^{j+1} C_{m}}{\partial z_{1}^{j+1}}\right. \\
& \left.-\frac{1}{P e^{2}} f_{j} \frac{\partial^{j+2} C_{m}}{\partial z_{1}^{j+2}}+f_{j} \frac{\partial^{j+1} C_{m}}{\partial t \partial z_{1}^{j}}+\alpha^{2} f_{j} \frac{\partial^{j} C_{m}}{\partial z_{1}^{j}}\right]=0 .
\end{aligned}
$$

\subsubsection{Generalized dispersion model}

Generalized dispersion model is one of the models that can describe the whole dispersion process, including the dispersion coefficient and how the dispersion process changes depending on time. Using the approach of Gill and Sankarasubramanian [6] by multiplying Eq. (20) by ' $2 r$ ' and integrating it with respect to $r$ from zero to $R(z)$ and using Eq. (31), one can get the generalized dispersion model for $C_{m}\left(z_{1}, t\right)$ on the dispersion of solute with chemical reaction as 
$\frac{\partial C_{m}}{\partial t}=\sum_{i=1}^{\infty} K_{i}(t) \frac{\partial^{i} C_{m}}{\partial z_{1}^{i}}\left(z_{1}, t\right)-\beta^{2} C_{m}\left(z_{1}, t\right)$,

where $K_{i}(t)$ is the dispersion coefficients. $K_{1}(t)$ is the coefficient of longitudinal convection and $K_{2}(t)$ is the coefficient of longitudinal diffusion. Since, the coefficient $K_{2}(t)$ expresses the whole dispersion process in terms of simple diffusion process; it is also called as the effective axial diffusivity. Since the value of the dispersion coefficient $K_{3}(t)$ for Newtonian fluid is $K_{3}(t \rightarrow \infty)=-1 / 23040$ which is negligibly small, the study ignored the terms $K_{3}(t), K_{4}(t)$ and so on [6]. Using Eq. (33) in Eq. (32) and grouping the coefficients of $\partial^{j} C_{m} / \partial z_{1}^{j}, j=1,2, \ldots$ together, yields

$$
\begin{gathered}
{\left[\frac{\partial f_{1}}{\partial t}-L^{2} f_{1}+u-u_{m}+K_{1}(t)+\beta^{2} f_{1}\right] \frac{\partial C_{m}}{\partial z_{1}}+\left[\frac{\partial f_{2}}{\partial t}-L^{2} f_{2}+\left(u-u_{m}\right) f_{1}+K_{1}(t) f_{1}+K_{2}(t)-\frac{1}{P e^{2}}+\beta^{2} f_{2}\right] \frac{\partial^{2} C_{m}}{\partial z_{1}^{2}}} \\
+\sum_{j=1}^{\infty}\left[\frac{\partial f_{j+2}}{\partial t}-L^{2} f_{j+2}+\left(u-u_{m}\right) f_{j+1}+\beta^{2} f_{j+2}-\frac{1}{P e^{2}} f_{j}+\sum_{i=1}^{j+1} K_{i}(t) f_{j+2-i}+K_{j+2}(t)\right] \frac{\partial^{j+2} C_{m}}{\partial z_{1}^{j+2}}=0 .
\end{gathered}
$$

From Eq. (34), by equating the coefficients of $\partial^{j} C_{m} / \partial z_{1}^{j}$ to zero for $j=1,2,3, \ldots$, the following infinite system of partial differential equations is given by

$$
\begin{aligned}
& \frac{\partial f_{1}}{\partial t}-L^{2} f_{1}+u-u_{m}+K_{1}(t)-\beta^{2} f_{1}=0, \\
& \frac{\partial f_{2}}{\partial t}-L^{2} f_{2}+\left(u-u_{m}+K_{1}(t)\right) f_{1}+K_{2}(t)-\frac{1}{P e^{2}}-\beta^{2} f_{2}=0, \\
& \frac{\partial f_{j+2}}{\partial t}-L^{2} f_{j+2}+\left(u-u_{m}+K_{1}(t)\right) f_{j+1}+\left(K_{2}(t)-\frac{1}{P e^{2}}\right) f_{j}-\beta^{2} f_{j+2}+\sum_{i=2}^{j+2} K_{i}(t) f_{j+2-i}=0,
\end{aligned}
$$

for $j=1,2, \ldots$ Since $C\left(r, z_{1}, t\right)$ is expressed in terms of $C_{m}\left(z_{1}, t\right)$ in Eq. (27), $C_{m}\left(z_{1}, t\right)$ can be chosen to satisfy the initial and boundary conditions of $C\left(r, z_{1}, t\right)$, then, Eq. (23)-(25) and Eq. (30) imply that the $f_{j}$ must satisfy the following initial and boundary conditions

$$
\begin{aligned}
& f_{j}(r, 0)=0, \\
& \frac{\partial f_{j}}{\partial r}(0, t)=0=\frac{\partial f_{j}}{\partial r}[R(z), t] .
\end{aligned}
$$

Substituting Eq. (30) into Eq. (31) yields the solvability condition given by

$$
\int_{0}^{R(z)} f_{j} r d r=0
$$

Multiplying Eq. (35) by $r$ and integrating the resulting equation between zero and $R(z)$ with respect to $r$ and use of the solvability condition (40), one can get 


$$
K_{1}(t)=-2 \int_{0}^{R(z)}\left(u-u_{m}\right) r d r=0 .
$$

Similarly, applying the same procedure in Eq. (36) and Eq. (37) to obtain

$$
\begin{aligned}
& K_{2}(t)=\frac{1}{P e^{2}}-2 \int_{0}^{R(z)} f_{1} u r d r, \\
& K_{j+2}(t)=-2 \int_{0}^{R(z)} f_{j+1} u r d r, j=1,2, \ldots
\end{aligned}
$$

\subsubsection{Solution of $f_{1}(r, t)$}

The dispersion function $f_{1}(r, t)$ is the coefficient of $\partial C_{m} / \partial z_{1}$ which plays an important role in measuring the deviation of the local concentration $C\left(r, z_{1}, t\right)$ from the mean concentration $C_{m}\left(z_{1}, t\right)$ . The solution of Eq. (35) satisfying the boundary conditions (38) and (39) can be divided into two states which is steady state and unsteady state. The expression of $f_{1}(r, t)$ is expressed in the form of

$$
f_{1}(r, t)=f_{1 s}(r)+f_{1 t}(r, t)
$$

where $f_{1 s}(r)$ is the dispersion function in the steady state and $f_{1 t}(r, t)$ is the dispersion function in the unsteady state that describes the time-dependent nature of the dispersion of the solute. Applying Eq. (44) in Eq. (35), yields

$$
\frac{\partial f_{1 s}}{\partial t}+\frac{\partial f_{1 t}}{\partial t}-L^{2}\left(f_{1 s}+f_{1 t}\right)+\left(u-u_{m}\right)+\beta^{2}\left(f_{1 s}+f_{1 t}\right)=0
$$

The term $\partial f_{1 s} / \partial t$ equal to zero for steady state dispersion. Grouping the $f_{1 t}(r, t)$ terms together and the other terms together, and equating each of these to zero, the simplified differential equations for $f_{1 s}(r)$ and $f_{1 t}(r, t)$ is given in the form of

$$
\begin{aligned}
& L^{2} f_{1 s}-\left(u-u_{m}\right)+\beta^{2} f_{1 s}=0, \\
& \frac{\partial f_{1 t}}{\partial t}=L^{2} f_{1 t}-\beta^{2} f_{1 t} .
\end{aligned}
$$

Substituting Eq. (44) in Eq. (38) and Eq. (39) and then applying the same procedure, it yields the following simplified boundary conditions for $f_{1 s}(r)$ and $f_{1 t}(r, t)$

$$
\begin{aligned}
& f_{1 t}(r, 0)=-f_{1 s}(r), \\
& \frac{d f_{1 s}}{d r}(r=0)=0=\frac{d f_{1 s}}{d r}(r=R(z)),
\end{aligned}
$$




$$
\frac{\partial f_{1 t}}{\partial r}(0, t)=0=\frac{\partial f_{1 t}}{\partial r}(R(z), t)
$$

Similarly, using Eq. (44) in the solvability condition (40), one can obtain the following useful condition

$$
\int_{0}^{R(z)} f_{1 t} r d r=-\int_{0}^{R(z)} f_{1 s} r d r=0
$$

Solving Eq. (46) subject to the condition (49) by using Mathematica program, the steady dispersion function in the plug flow region $f_{1 s_{-}}(r)$ is given by

$$
\begin{aligned}
& f_{1 s_{-}}(r)=-\frac{1}{\beta^{2}}\left(\frac{2}{(n+3)}+\frac{2(n+1)}{(n+2) R(z)} r_{p}+\frac{n}{[R(z)]^{2}} r_{p}^{2}-\frac{n(n-1)}{2[R(z)]^{n+1}} r_{p}^{n+1}+\frac{\left(n^{4}+2 n^{3}-5 n^{2}-6 n+4\right)}{2(n+2)(n+3)[R(z)]^{n+3}} r_{p}^{n+3}\right) \\
& +C I J_{0}\left(i \beta r_{p}\right) \text { if } 0 \leq r \leq r_{p},
\end{aligned}
$$

where $J_{0}$ is the first kind Bessel's functions of order zero and $C l$ is a constant integration. The steady dispersion function in the outer flow region for $f_{1 s+}(r)$ if $r_{p} \leq r \leq R(z)$ is not shown in this paper because of the complexity and long expressions. Solving Eq. (47) by using the variable separable method subject to the boundary conditions (48), (50) and (51), the solution of $f_{1 t}(r, t)$ is obtained as

$$
f_{1 t}(r, t)=e^{-\beta^{2} t} \sum_{m=1}^{\infty} A_{m} e^{-\lambda_{m}^{2} t} J_{0}\left(\lambda_{m} r\right)
$$

where $\lambda_{m}$ 's denotes the root of the equation $J_{1}\left(\lambda_{m}\right)=0$, where $J_{1}$ is the Bessel's functions of the first kind of order one and

$$
A_{m}=-\frac{\int_{0}^{R(z)} J_{0}\left(\lambda_{m} r\right) f_{1 s}(r) r d r}{\int_{0}^{R(z)} J_{0}^{2}\left(\lambda_{m} r\right) r d r}=-\frac{2}{J_{0}^{2}\left(\lambda_{m}\right)} \int_{0}^{R(z)} J_{0}\left(\lambda_{m} r\right) f_{1 s}(r) r d r=-\frac{2}{J_{0}^{2}\left(\lambda_{m}\right)}\left(I_{1}+I_{2}\right) .
$$

The expression of $I_{1}$ and $I_{2}$ in Eq. (54) are given by

$$
\begin{aligned}
& I_{1}=\int_{0}^{r_{p}} J_{0}\left(\lambda_{m} r\right) f_{1 s_{-}}(r) r d r, \\
& I_{2}=\int_{r_{p}}^{R(z)} J_{0}\left(\lambda_{m} r\right) f_{1 s_{+}}(r) r d r .
\end{aligned}
$$

Then, the integrals in Eq. (55) and Eq. (56) have been evaluated using Mathematica program to get the data for plotting the graphs of dispersion function for unsteady part. 


\subsubsection{Solution of longitudinal diffusion coefficient $K_{2}(t)$}

It is to be noted that $K_{2}(t)$ is a very useful dispersion coefficient appearing in the generalized dispersion model represented by Eq. (42). It is used to measure the rate of solute dispersion in the fluid. Using Eq. (26), Eq. (27), Eq. (44), Eq. (52)-(56) in Eq. (42), the expression for the longitudinal diffusion coefficient $K_{2}(t)$ has been obtained using Mathematica program. The expression of $K_{2}(t)$ is not shown in this paper because too long. Once $K_{2}(t)$ is known, $f_{2}(r, t)$ can be obtained from Eq. (36) by using similar method that was applied to get $f_{1}(r, t)$. Substitution of the expression of $f_{2}(r, t)$ in Eq. (37) yields $K_{3}(t)$ and proceeding in a similar way, one can find $f_{3}(r, t), K_{4}(t), f_{4}(r, t), K_{5}(t)$ and so on from Eq. (37) and Eq. (43), recursively. Since the value of the dispersion coefficient $K_{3}(t)$ is negligibly small and the solutions obtained for $f_{1}(r, t)$ and $K_{2}(t)$ are very lengthy and highly complicated, the computations of $K_{j+2}$ and $f_{j+1}(r, t)$ for $j=1,2, \ldots$ have been neglected.

\section{Results and Discussions}

The present study goals to investigate the influences of stenosis and chemical reaction on the velocity of $\mathrm{H}-\mathrm{B}$ fluid, solute concentration and dispersion coefficient by the varying the parameter of stenosis height, stenosis length, artery radius in the radial direction $r$ and plug flow radius $r_{p}$. The present results of $\mathrm{H}-\mathrm{B}$ fluid with the effect of stenosis and chemical reaction in bloodstream are beneficial to determine the effectiveness of solute dispersion in a narrow stenosed artery and also important to determine the rate of reaction between the blood protein and solute. The range of values of parameters used in this study are as follows: yield stress $r_{p}: 0-0.2$; power-law index $n: 0.95$ 1.05; rate of chemical reaction parameter $\beta: 0-8[13,14]$. The present results of dispersion coefficient in $\mathrm{H}-\mathrm{B}$ fluid with the influence of stenosis and chemical reaction has been validated with Jaafar [10] by letting the stenosis height and chemical reaction parameter equal to zero.

The validation of steady dispersion function $f_{1 s}$ and unsteady dispersion function $f_{1 t}$ when $\delta=0$, $\beta=0.1, n=0.95, t=0.1$ and $r_{p}=0.1$ are illustrated in Figure 2 and Figure 3 . It is depicted that the dispersion function without stenosis in the present study are in good agreement with the dispersion function shown in Jaafar [10]. In the present study, when the stenosis height is absence $(\delta=0)$, the radius of artery at the stenosed region is $R(z)=1$. 


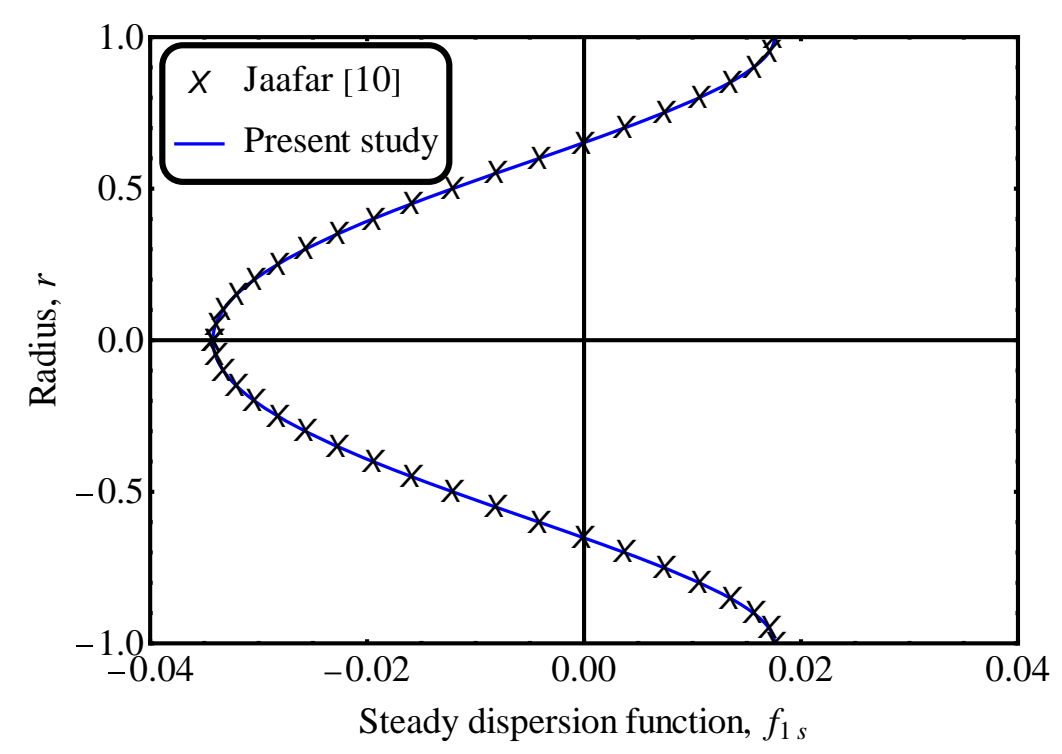

Fig. 2. Steady dispersion function $f_{1 s}$ with radius $r$ when $\delta=0$, $R(z)=1, \beta=0.1, n=0.95$ and $r_{p}=0.1$

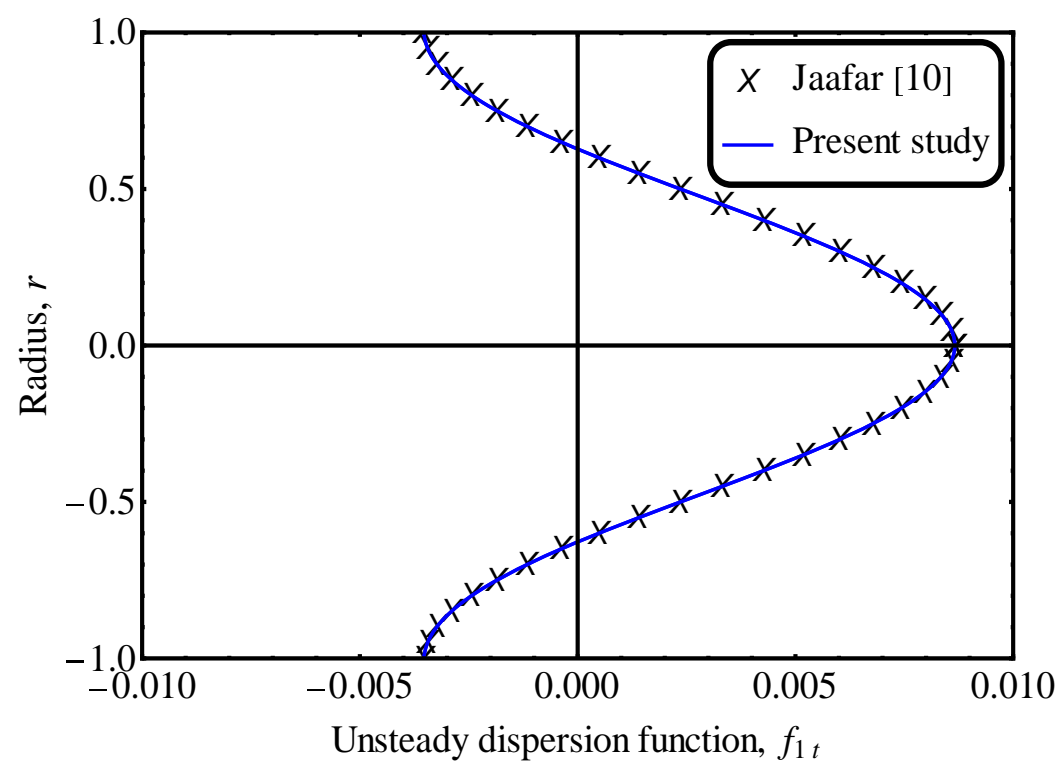

Fig. 3. Unsteady dispersion function $f_{1 t}$ with radius $r$ when $\delta=0$, $R(z)=1, \beta=0.1, t=0.1, n=0.95$ and $r_{p}=0.1$

\subsection{Steady Dispersion Function $f_{1 s}$}

Figure 4 shows that the variation of steady dispersion function $f_{1 s}$ with a radius $r$ for different values of chemical reaction when $l_{0}=3, d=2, z=4, \delta=0.1 r_{p}=0.1$ and $n=0.95$. It can be seen that when the chemical reaction increases, the steady dispersion function $f_{1 s}$ increases at the center of an artery and decreases at the wall of the inner artery. When the chemical reaction increases, the amount of free solute is low, and thus the velocity and solute dispersion at the center increases rapidly meanwhile it reduces the solute dispersion at the wall. 


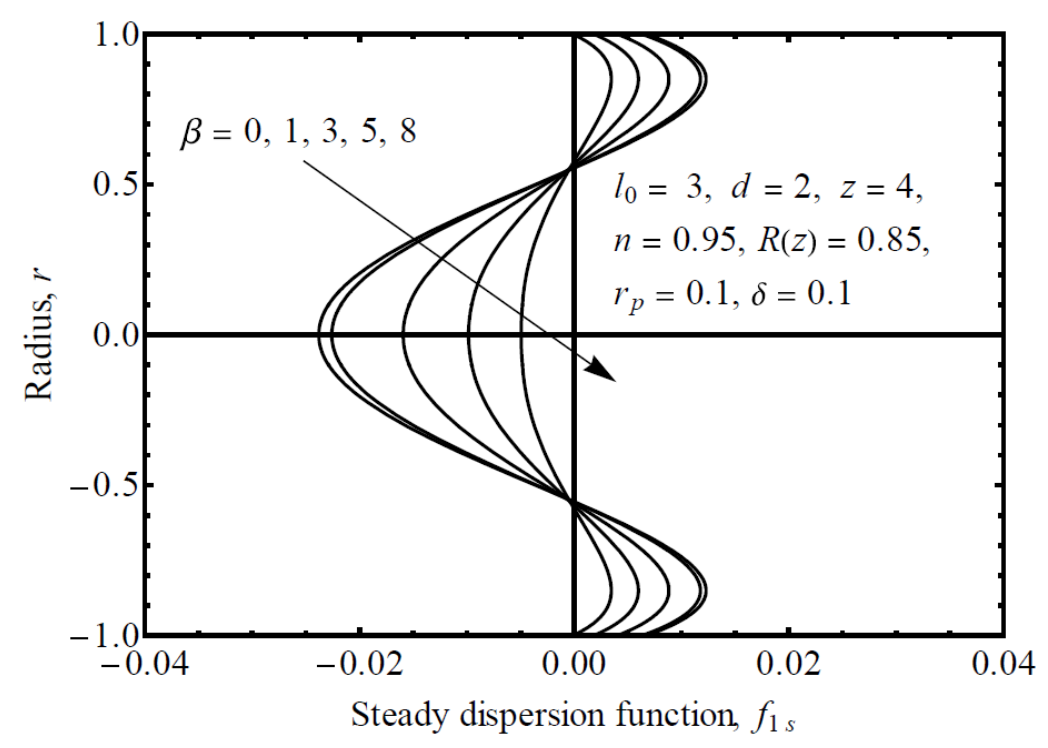

Fig. 4. Variation of steady dispersion function $f_{1 s}$ with a radius $r$ for different values of chemical reaction when $l_{0}=3, d=2, z=4, \delta=0.1 r_{p}=0.1$ and $n=0.95$.

Figure 5 depicts the variation of steady dispersion function $f_{1 s}$ with a radius $r$ for different values of stenosis height, $\delta$ when $l_{0}=3, d=2, z=4, r_{p}=0.1, \beta=0.1$ and $n=0.95$. The height of the stenosis has a significant impact on the size of the stenosis and affects the blood velocity and dispersion process. From the figure, it shows that at the centre of artery, the steady dispersion function of the solute increases as the stenosis height increases and the reverse behaviour occurs at the inner wall of artery. At the center, the region at stenosed artery becomes thinner as the stenosis height increase and the velocity of blood increases rapidly together with the solute, hence it increases the dispersion function.

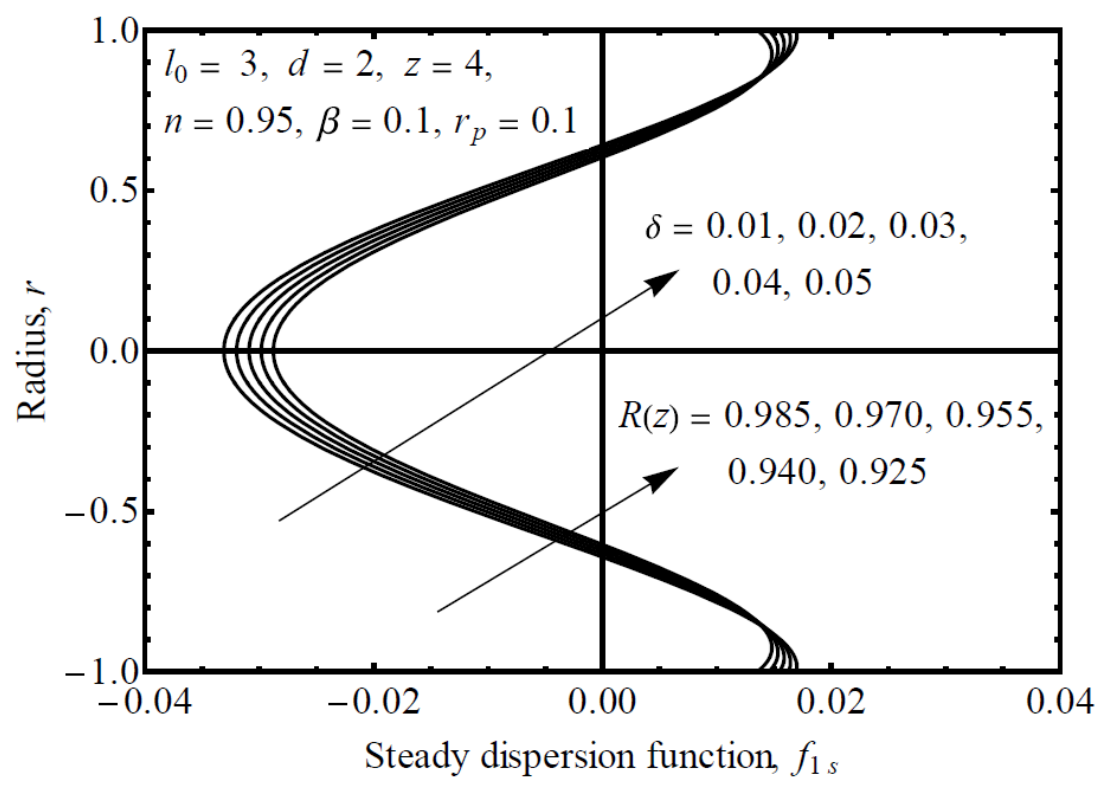

Fig. 5. Variation of steady dispersion function with a radius $r$ for different values of stenosis height, $\delta$ when $l_{0}=3, d=2, z=4$, $r_{p}=0.1, \beta=0.1$ and $n=0.95$ 


\subsection{Unsteady Dispersion Function $f_{1 t}$}

Variation of unsteady dispersion function for different values of chemical reaction rate parameter, $\beta$ when $l_{0}=3, d=2, z=4, r_{p}=0.2, t=0.2$ and $n=1.05$ is represented in Figure 6 . It is observed that when the chemical reaction rate parameter $\beta$ increases, the dispersion function $f_{1 t}$ slightly decreases at the center of artery between the range of the radius $r=-0.6$ to $r=0.6$ and it displays the reverse behavior at the inner wall of the artery between the range of the radius $r=-1$ to $r=-0.6$ and $r=0.6$ to $r=1$. It is due to the fact that the chemical reaction of blood protein and solute reacts at the early unsteady stage when $t=0.2$. It is observed that there is a high quantity of red blood cells at the center of the artery and a greater red blood cell aggregation, the greater chemical reaction occurs and thus the degree of the binding of solute to the blood proteins is high at the center. Then, there is smaller amount of solute are free and hence less effects near the wall. Therefore, the dispersion function increases when the chemical reaction increases at the center and reduces near the wall.

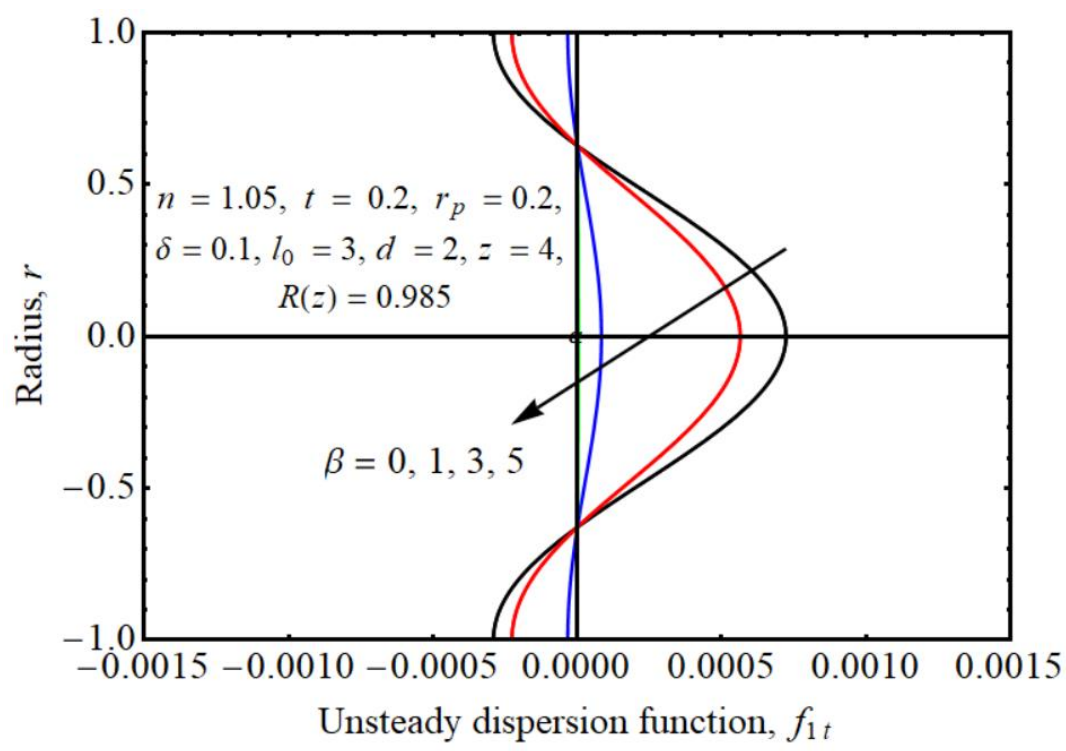

Fig. 6. Variation of unsteady dispersion function with a radius $r$ for different values of chemical reaction when $l_{0}=3, d=2, z=4$, $r_{p}=0.2, t=0.2$ and $n=1.05$

Variation of unsteady dispersion function for different values of stenosis height, $\delta$ when $\beta=0.1, l_{0}=3, d=2, z=4, n=0.95, t=0.1$ and $r_{p}=0.1$ is shown in Figure 7 . The effect of stenosis height is vital to identify the size of stenosis at the artery wall to investigate the dispersion process. It is observed that when the stenosis height increases in the range $\delta=0.01,0.02,0.03,0.04$ and 0.05 , it results in a decrease of the unsteady dispersion function $f_{1 t}$ of the solute due to the reduction of the viscosity in the blood. As the stenosis height increases, the region at stenosed artery becomes reduce and affects the normal flow of blood to the artery. 


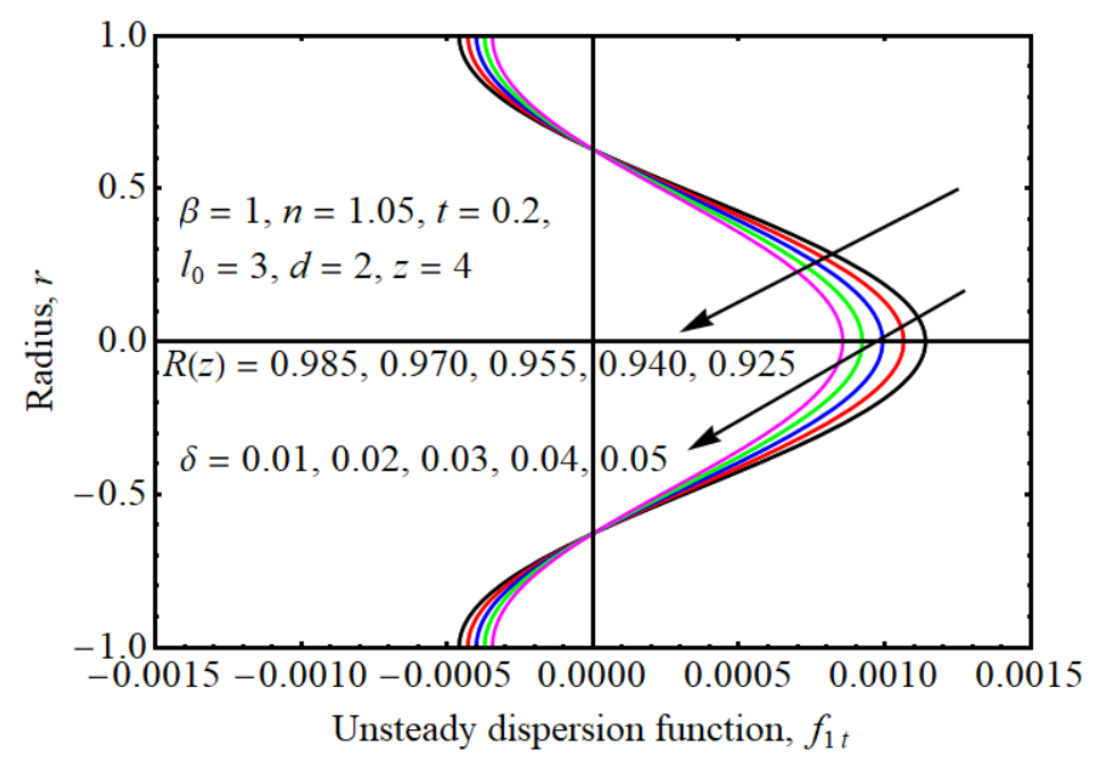

Fig. 7. Variation of unsteady dispersion function with a radius $r$ for different values of stenosis height, $\delta$ when $l_{0}=3, d=2, z=4$, $r_{p}=0.1, \beta=0.1, t=0.1$ and $n=0.95$

Figure 8 depicts the variation of unsteady dispersion function for different values of time when $\beta=1, n=1.05, \delta=0.01, l_{0}=3, d=2, z=4$ and $R(z)=0.985$. The dispersion function at unsteady state varies since the time is changed while the other parameter is held constant. As the time increases from $t=0$ to $t=0.5$, the dispersion function at unsteady state decreases significantly. At time $t=0$, the dispersion function is higher and after some time, the solute starts to decrease slowly due to the solute start to diffuse and occupy the surface area of artery and hence reduces the dispersion process. When $t=0.5$, the dispersion function tends to almost constant and zero values, and there is no change when radius of artery varies. It is noted that the dispersion function decreases at the center with the increase of the plug core radius and vice versa happens at the wall of the artery. As observed by Patel and Sirs [15], one of the factors that influences the dispersion of solutes in blood flow is the aggregation of red blood cells and their migration to the center of artery. The aggregation of red blood cells is considered to significantly disrupt blood flow in an artery and enhance vascular flow resistance.

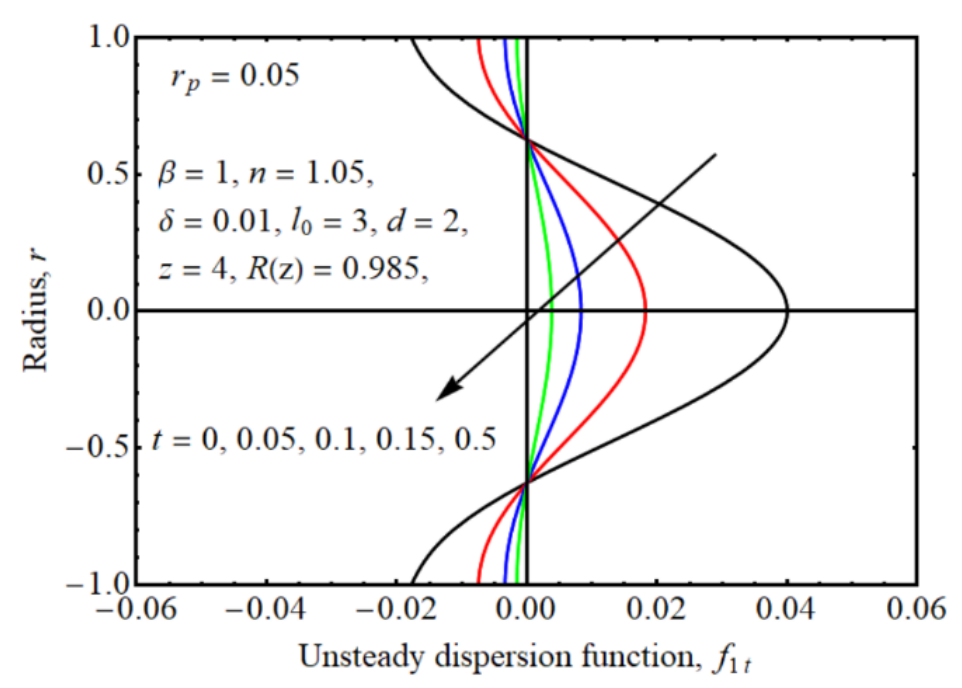

(a) 


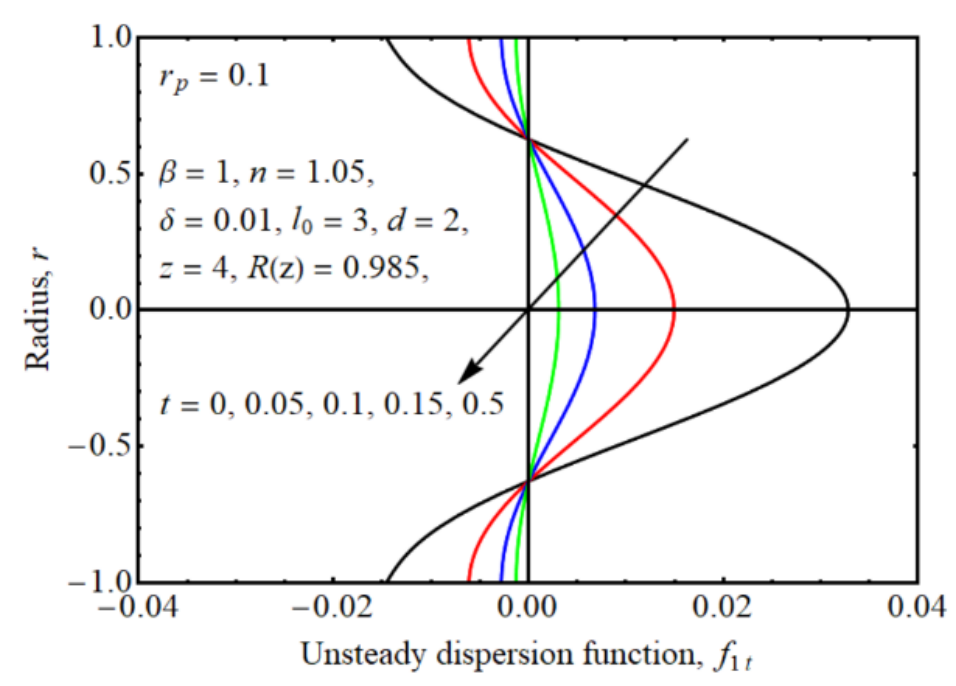

(b)

Fig. 8. Variation of unsteady dispersion function with a radius $r$ for different values of times when $\beta=1, n=1.05, \delta=0.01, l_{0}=3, d=2, z=4$ and, $R(z)=0.985$ at (a) $r_{p}=0.05$ and (b) $r_{p}=0.1$

\section{Conclusion}

This mathematical analysis brings out many interesting and useful results on the dispersion of a solute in the steady flow of blood modeled as a Herschel-Bulkley fluid. The findings of these studies are compared with the results of Jaafar [10] in the absence of stenosis. The major findings are summarized as below

i. It is observed that the dispersion coefficient which describes the entire diffusion phenomenon is changed due to the non-Newtonian behavior of the fluid which is the yield stress and power-law index and also due to the presence of stenosis in the wall of the artery.

ii. The unsteady dispersion function decreases at the center of an artery with the increase of the stenosis height and chemical reaction. Meanwhile the steady dispersion function increases at the center of an artery with the increase of the stenosis height and chemical reaction.

iii. It is noted that there is a high amount of red blood cells at the center of the artery but less influences near the wall hence, the degree of the binding of solute to the blood proteins is high at the center and fewer molecules of solute are free. As a result, as the chemical reaction rate parameter, plug core radius and time increase, the dispersion function decreases at the center of an artery.

From the results obtained in this study, we observe that the present H-B fluid model may be useful in predicting the physiologically important flow quantities with better accuracy. It is concluded that the present mathematical analysis may be considered as an improvement in the mathematical modeling of unsteady dispersion of solutes in blood flow through narrow stenosed arteries with the presence of chemical reaction. Since the presence of stenosis in the wall of an artery will alter the flow of blood or blocked the blood and oxygen to the heart, the study on the stenosed artery would be helpful to cater to this problem.

In this study, the research investigates the problem on the solute dispersion in blood flow analytically. Since blood flow is extremely unsteady in nature, it will be beneficial in further research to investigate the effect of unsteady flow on solute dispersion in stenosed artery. This study also strictly limited to the analytical solution. The results could be more useful to further the study by 
using Computational Fluid Dynamics approach and contribute well to the observations of the experiment [16]. The studies can be carried out by investigating the effect of heat transfer on the dispersion of solute numerically $[17,18]$.

\section{Acknowledgments}

The authors would like to acknowledge Research Management Centre-UTM, Universiti Teknologi Malaysia for the financial support through vote numbers Q. J130000.2654.17J12 (GUP Tier 2 Grant). The valuable suggestions of the reviewers which led to the improvement of the manuscript are greatly appreciated.

\section{References}

[1] Ali, N., A. Zaman, M. Sajid, J. J. Nieto, and A. Torres. "Unsteady non-Newtonian blood flow through a tapered overlapping stenosed catheterized vessel." Mathematical Biosciences 269 (2015): 94-103. https://doi.org/10.1016/i.mbs.2015.08.018

[2] Jaafar, Nurul Aini, Yazariah Mohd Yatim, and D. S. Sankar. "Influence of chemical reaction on the steady dispersion of solute in blood flow-a mathematical model." Far East Journal of Mathematical Sciences 100, no. 4 (2016): 617. https://doi.org/10.17654/MS100040617

[3] Taylor, Geoffrey Ingram. "Dispersion of soluble matter in solvent flowing slowly through a tube." Proceedings of the Royal Society of London. Series A. Mathematical and Physical Sciences 219, no. 1137 (1953): $186-203$. https://doi.org/10.1098/rspa.1953.0139

[4] Aris, Rutherford. "On the dispersion of a solute in a fluid flowing through a tube." Proceedings of the Royal Society of London. Series A. Mathematical and Physical Sciences 235, no. 1200 (1956): 67-77. https://doi.org/10.1098/rspa.1956.0065

[5] Ananthakrishnan, Vs, Wr N. Gill, and Allen J. Barduhn. "Laminar dispersion in capillaries: Part I. Mathematical analysis." AIChE Journal 11, no. 6 (1965): 1063-1072. https://doi.org/10.1002/aic.690110620

[6] Gill, W. N., and R. Sankarasubramanian. "Exact analysis of unsteady convective diffusion." Proceedings of the Royal Society of London. A. Mathematical and Physical Sciences 316, no. 1526 (1970): 341-350. https://doi.org/10.1098/rspa.1970.0083

[7] Dash, R. K., G. Jayaraman, and K. N. Mehta. "Shear augmented dispersion of a solute in a Casson fluid flowing in a conduit." Annals of Biomedical Engineering 28, no. 4 (2000): 373-385. https://doi.org/10.1114/1.287

[8] Rana, Jyotirmoy, and P. V. S. N. Murthy. "Unsteady solute dispersion in Herschel-Bulkley fluid in a tube with wall absorption." Physics of Fluids 28, no. 11 (2016): 111903. https://doi.org/10.1063/1.4967210

[9] Rana, Jyotirmoy, and P. V. S. N. Murthy. "Unsteady solute dispersion in small blood vessels using a two-phase Casson model." Proceedings of the Royal Society A: Mathematical, Physical and Engineering Sciences 473, no. 2204 (2017): 20170427. https://doi.org/10.1098/rspa.2017.0427

[10] Jaafar, Nurul Aini. "Mathematical Analysis of Herschel-Bulkley Fluid Model for Solute Dispersion in Blood Flow Through Narrow Conduits." PhD thesis, Universiti Sains Malaysia, 2017.

[11] Rana, Jyotirmoy, and P. V. S. N. Murthy. "Unsteady solute dispersion in Herschel-Bulkley fluid in a tube with wall absorption." Physics of Fluids 28, no. 11 (2016): 111903. https://doi.org/10.1063/1.4967210

[12] Jaafar, Nurul Aini, Yazariah Mohd Yatim, and D. S. Sankar. "Mathematical analysis for unsteady dispersion of solute with chemical reaction in blood flow." In AIP Conference Proceedings, vol. 1750, no. 1, p. 030033. AIP Publishing LLC, 2016. https://doi.org/10.1063/1.4954569

[13] Miah, Md. Abdul Karim, Shorab Hossain, and Sayedus Salehin. "Effects of Severity and Dominance of Viscous Force on Stenosis and Aneurysm During Pulsatile Blood Flow Using Computational Modelling." CFD Letters 12, no. 8 (2020): 35-54. https://doi.org/10.37934/cfdl.12.8.3554

[14] Ratchagar, Nirmala P., and R. Vijaya Kumar. "Dispersion of solute with chemical reaction in blood flow." Bulletin of Pure \& Applied Sciences-Mathematics and Statistics 38, no. 1 (2019): 385-395. https://doi.org/10.5958/2320$\underline{3226.2019 .00042 .0}$

[15] Patel, I. C., and J. A. Sirs. "Dispersion of solutes during blood flow through curved tubes." Medical and Biological Engineering and Computing 21, no. 2 (1983): 113-118. https://doi.org/10.1007/BF02441524

[16] Al-Azawy, Mohammed Ghalib, Saleem Khalefa Kadhim, and Azzam Sabah Hameed. "Newtonian and NonNewtonian Blood Rheology Inside a Model of Stenosis." CFD Letters 12, no. 11 (2020): 27-36. https://doi.org/10.37934/cfdl.12.11.2736 
[17] Sahak, Ahmad Sofianuddin A., Nor Azwadi Che Sidik, Siti Nurul Akmal Yusof, and Mahmoud Ahmed Alamir. "Numerical Study of Particle Behaviour in a Mixed Convection Channel Flow with Cavity using Cubic Interpolation Pseudo-Particle Navier-Stokes Formulation Method." Journal of Advanced Research in Numerical Heat Transfer 1, no. 1 (2020): 32-51.

[18] Ewis, Karem Mahmoud. "Effects of Variable Thermal Conductivity and Grashof Number on Non-Darcian Natural Convection Flow of Viscoelastic Fluids with Non Linear Radiation and Dissipations." Journal of Advanced Research in Applied Sciences and Engineering Technology 22, no. 1 (2021): 69-80. 